\title{
The Design Methodology of Fuzzy Controller Based on Information Granulation (IG)-Based Optimization Approach
}

\author{
Sung-Kwun $\mathrm{Oh}^{1}$, Seok-Beom Roh ${ }^{1}$, and Dong-Yoon Lee ${ }^{2}$ \\ ${ }^{1}$ School of Electrical, Electronic and Information Engineering, Wonkwang University, South \\ Korea \\ \{ohsk, nado\}@wonkwang.ac.kr \\ 2 Department of Information and Communication Engineering, Joongbu University, \\ South Korea \\ dylee@joongbu.ac.kr
}

\begin{abstract}
In this study, we present information granulation (IG)-based optimization approach to the design of fuzzy controller. The design procedure dwells on the use of evolutionary computing (genetic algorithms) and the estimation of the polynomial model that is carried out by effectively combining Hard CMeans Clustering (HCM) Method with Least Mean Square (LSM) method. The developed approach is applied to a nonlinear system such as an inverted pendulum where we show the results of comprehensive numerical studies and carry out a detailed comparative analysis
\end{abstract}

\section{Introduction}

Recently, the ongoing challenge we are facing when design advanced system control has resulted in a diversity of underlying methodologies, development platforms and detailed algorithms. As one of the representative design approaches, a design approach of fuzzy controller by means of IG-based estimation technique is introduced in this study. The intent of this study is to develop, optimize and experiment with the fuzzy controllers when developing a general design scheme of Computational Intelligence. One of the difficulties in the construction of the fuzzy controller is to derive a set of optimal control parameters of the controller such as linguistic control rules, scaling factors, and membership functions of the fuzzy controller[7,8]. Genetic algorithms (GAs) can be used to find the optimal control parameters. However, evolutionary computing is computationally intensive and this may be a point of concern when dealing with amount of time available to such search. For instance, when controlling a nonlinear plant such as an inverted pendulum of which initial states vary in each case, the search time required by GAs could be prohibitively high when dealing with dynamic systems. As a consequence, the parameters of the fuzzy controller cannot be easily adapted to the changing initial states of this system such as an angular position 
and an angular velocity of the pendulum. To alleviate this shortcoming, we introduce a nonlinear mapping such as HCM-LMS tandem based polynomial. The development process consists of two main phases. First, using genetic optimization we determine optimal parameters of the fuzzy controller for various initial states (conditions). Second, we build up a nonlinear model that captures a relationship between the initial states of the system and the corresponding genetically optimized control parameters.

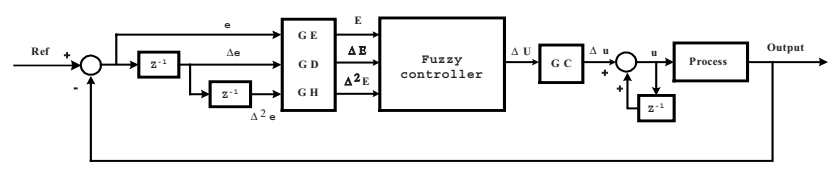

Fig. 1. An overall architecture of the fuzzy PID controller

\section{Design Methodology of Fuzzy Controller}

The block diagram of fuzzy PID controller is shown in Figure $1[5,6]$.

\subsection{Auto-tuning of the Fuzzy Controller by Gas}

Genetic algorithms (GAs) are the search algorithms inspired by nature in the sense that we exploit a fundamental concept of a survival of the fittest as being encountered in selection mechanisms among species[1]. The overall design procedure of the fuzzy PID controller realized by means of GAs consists of the following steps

[Step 1.] Select the general structure of the fuzzy controller according to the purpose of control and dynamics of the process.

[Step 2.] We set the initial individuals of GAs for the scaling factor of fuzzy controller.

[Step 3.] Here, all the control parameters such as the scaling factors GE, GD, GH and $\mathrm{GC}$ are tuned at the same time.

\subsection{HCM-LMS Tandem Based Polynomial Model}

Step1: Fix $c(2 \leq c<n)$ and initialize the partition matrix $\mathbf{U}^{(0)} \in M_{C}$

Step 2: Calculate the center vectors $\mathbf{V}_{i}$ of each cluster :

$$
v_{i j}^{(r)}=\sum_{k=1}^{n} u_{i k}^{(r)} \cdot x_{k j} / \sum_{k=1}^{n} u_{i k}^{(r)}
$$

Step 3: Update the partition matrix $\mathrm{U}^{(\mathrm{r})}$

$$
d_{i k}=d\left(\mathbf{x}_{k}-\mathbf{v}_{i}\right)=\left\|\mathbf{x}_{k}-\mathbf{v}_{i}\right\|=\left[\sum_{j=1}^{m}\left(x_{k j}-v_{i j}\right)^{2}\right]^{1 / 2}
$$




$$
u_{i k}^{(r+1)}=\left\{\begin{array}{cc}
1 & d_{i k}^{(r)}=\min \left\{d_{j k}^{(r)}\right\} \text { for all } j \in c \\
0 & \text { otherwise }
\end{array}\right.
$$

Step 4: Check a termination criterion. If

$$
\left\|\mathrm{U}^{(\mathrm{r}+1)}-\mathrm{U}^{(\mathrm{r})}\right\| \leq \varepsilon(\text { tolerance level })
$$

stop ; otherwise set $r=r+1$ and return to step 2[2][3].

\section{Experimental Studies}

In this section, we demonstrate the effectiveness of the fuzzy PID controller by applying it to the inverted pendulum system. The control goal is to balance the pole starting from nonzero conditions by supplying appropriate force to the cart[4].

First, we select several initial angular positions and angular velocity and obtain the auto-tuned control parameters by means of GAs according to the change of each selected initial angular positions and angular velocity, then build a table. Secondly, we use HCM-LMS tandem-based polynomial to estimate the control parameters in the case that the initial angular positions and angular velocity of the inverted pendulum are selected arbitrarily within the given range. Table 1 shows the estimated scaling factors of the fuzzy PID controller and describes performance index of the fuzzy PID controller with the estimated scaling factors. Figure 1 demonstrates pole angle for (a) Case 1 and (b) Case 2 respectively

Table 1. The estimated parameters by means of the HCM-LMS tandem based polynomial and performance index(ITAE, Overshoot $(\%)$ ) of the fuzzy PID controller

\begin{tabular}{|c||c||c||c||c||c||c||c||c||c|}
\hline \multicolumn{1}{|c||}{ Case } & $\theta$ & $\dot{\theta}$ & GE & GD & GH & GC & ITAE & OS(\%) & Rising Time(sec) \\
\hline \hline 1 & 0.22 & 0.22 & 2.951133 & 60.959881 & 243.663513 & 1.770746 & 0.169122 & 0.083650 & 0.145199 \\
\hline \hline 2 & 0.22 & 0.45 & 1.713128 & 63.498253 & 246.882355 & 1.886055 & 0.634893 & 0.000000 & 0.322975 \\
\hline \hline 3 & 0.22 & 0.78 & 2.600188 & 59.980007 & 269.163086 & 2.219068 & 0.285220 & 0.529590 & 0.169282 \\
\hline \hline 4 & 0.45 & 0.22 & 1.706843 & 42.527325 & 106.950111 & 6.492052 & 0.882041 & 0.000000 & 0.233517 \\
\hline \hline 5 & 0.45 & 0.45 & 1.789289 & 63.856773 & 84.001183 & 6.829825 & 1.267673 & 0.000000 & 0.285800 \\
\hline \hline 6 & 0.45 & 0.78 & 1.918489 & 61.384880 & 245.082535 & 3.747335 & 0.960818 & 0.000000 & 0.193290 \\
\hline \hline 7 & 0.78 & 0.22 & 1.427422 & 36.951550 & 174.614471 & 4.790281 & 2.421195 & 1.954839 & 0.209058 \\
\hline \hline 8 & 0.78 & 0.45 & 1.366799 & 39.255482 & 190.132248 & 3.867553 & 2.806530 & 1.624381 & 0.210987 \\
\hline \hline 9 & 0.78 & 0.78 & 1.290725 & 38.158276 & 181.288132 & 3.872698 & 3.501827 & 0.574551 & 0.217495 \\
\hline
\end{tabular}

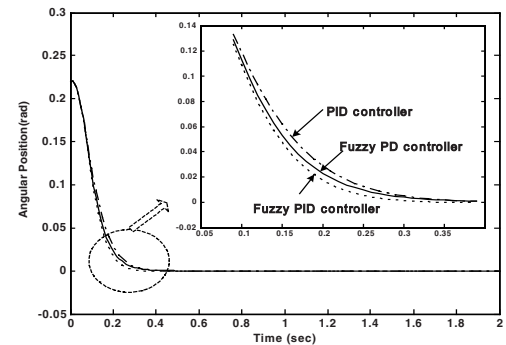

(a)

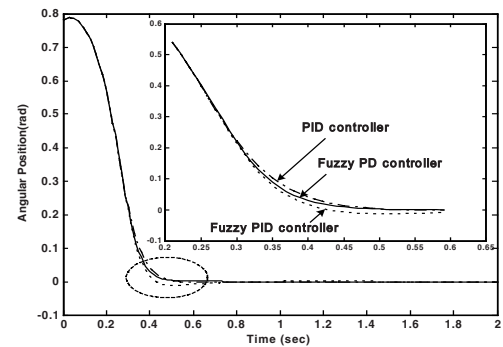

(b)

Fig. 2. Pole angle for (a) Case 1 and (b) Case 2 
From the above Figures 2, we observe that the fuzzy PD and fuzzy PID controllers control effectively the inverted pendulum system. The proposed estimation algorithm such as HCM-LMS tandem-based polynomial generates the preferred model architecture.

\section{Conclusion}

In this paper, we have proposed a two-phase optimization scheme of the fuzzy PID and PD controllers. The parameters under optimization concern scaling factors of the input and output variables of the controller that are known to exhibit an immense impact on its quality. The first phase of the design of the controller uses genetic computing that aims at the global optimization of its scaling factors where they are optimized with regard to a finite collection of initial conditions of the system under control. In the second phase, we construct a nonlinear mapping between the initial conditions of the system and the corresponding values of the scaling factors. From the simulation studies, using genetic optimization by scaling factor estimation modes and the estimation algorithm of the HCM-LMS tandem based polynomial model, we showed that the fuzzy PD/PID controller controls effectively the inverted pendulum system in a nonlinear range of $\theta$.

Acknowledgement. This work has been supported by EESRI(R-2003-0-285), which is funded by MOCIE (Ministry of commerce, industry and energy)

\section{References}

1. D.E. Goldberg: Genetic algorithms in Search, Optimization, and Machine Learning. Addison-Wesley (1989)

2. H.-S. Park, S.-K. Oh: Multi-FNN identification Based on HCM Clustering and Evolutionary Fuzzy Granulation. International Journal of Control, Automation, and Systems. 1(2) (2003) 194-202

3. J. Q. Chen, Y. G. Xi, Z.J. Zhang: A clustering algorithm for fuzzy model identification. Fuzzy Sets and Systems. 98 (1998) 319-329

4. J.R. Jang: Self-Learning Fuzzy Controllers Based on Temporal Back Propagation. IEEE Trans. On Neural Networks. 3(1992) 714-723

5. T.j. Procyk, E.H. Mamdani: A linguistic synthesis of fuzzy controller. Automatica. 15 (1979) 15-30

6. H. X. Li: A comparative design and tuning for conventional fuzzy control. IEEE Trans. Syst., Man, Cybern. B. 27 (1997) 884-889

7. S.-K. Oh, W. Pedrycz: The Design of Hybrid Fuzzy Controllers Based on Genetic Algorithms and Estimation Techniques. Kybernetes 31(2002) 909-917

8. S.K. Oh, T. Ahn, H. Hwang, J. Park, K. Woo: Design of a Hybrid Fuzzy Controller with the Optimal Auto-tuning Method. Journal of Control, Automation and Systems Engineering. 1 (1995) 63-70 